\title{
Atrophy of myelinated nerve fibres in the retina in optic neuritis
}

\author{
JAMES A. SHARPE* AND MICHAEL D. SANDERS \\ From the Department of Neuro-ophthalmology, \\ The National Hospital, Queen Square, London, W.C.I
}

Recognition of retinal nerve fibres is enhanced using bright red-free illumination with direct ophthalmoscopy or fundus photography (Vogt, 1913, 1921; Ito, Ozawa, Suga, and Mizuno, 1969; Hoyt, Rios-Montenegro, Behrens, and Eckelhoff, 1972a). Recently Hoyt and his co-workers have applied this technique to the clinical detection of axonal degeneration in the retina. Focal and diffuse patterns of retrograde nerve fibre atrophy have been described with optic neuritis (Hoyt, Schlicke, and Eckelhoff, 1972b) and glaucoma (Hoyt, Frisén, and Newman, 1973). Loss of retinal axons may be a sensitive indicator of optic nerve demyelination (Frisén and Hoyt, 1975).

Patches of medullated nerve fibres are occasionally present in the human retina, and this provides a unique opportunity to observe demyelination of the central nervous system in vivo. Disappearance of retinal myelin has previously been reported in optic atrophy with neurosyphilis (Wagenmann, 1894), pituitary tumour (Sachsalber, I905), central retinal artery embolism (Bachmann, I922), and glaucoma (Fuchs, 1951).

This report describes a patient with multiple sclerosis in whom focal loss of myelinated retinal nerve fibres occurred from retrograde axonal degeneration.

\section{Case history}

A 30-year-old Caucasian woman was referred to the National Hospital under the care of Dr. William Gooddy in June, 1974, for investigation of progressive bilateral visual impairment. At the ages of 18 and 21 years she had episodes of vertigo precipitated by extension of her neck. In June, 1972, she developed mild blurring of vision in both eyes and horizontal diplopia. An ophthalmologist found a divergent strabismus and corrective surgery was performed. Vision progressively deteriorated in both eyes during the next year. She complained of giddiness and

Address for reprints: M. D. Sanders, F.R.C.S., as above

* Supported in part by an Ontario Ministry of Health Fellowship and the Division of Neurology Toronto Western Hospital unsteadiness of gait when looking upwards. There was intermittent numbness of both legs since January, 1973. The patient was admitted to another hospital in January, 1974. Visual acuity was found to be $6 / 24$ in the right eye and $6 / 60$ in the left. An air encephalogram was normal. The cerebrospinal fluid contained $20 \mathrm{mg}$. protein per roo $\mathrm{ml}$. and the IgG level was not determined.

When she was examined at the National Hospital, cranial nerve function was normal apart from the visual and ocular motor findings. Tendon reflexes were increased in the arms and legs and there was sustained bilateral patellar and ankle clonus. Both plantar responses were extensor and abdominal reflexes were absent. Pain and light touch sensations were diminished in the lower legs.

Visual acuity was $4 / 60$ in the right eye and $5 / 60$ in the left eye. The pupils were $6 \mathrm{~mm}$. with diminished light responses. There was rebound nystagmus to the right and left and gaze-evoked nystagmus during upward gaze. Radiological investigations, including an EMI scan and vertebral angiography, were normal. Multiple sclerosis was diagnosed.

\section{FUNDOSCOPIC FINDINGS}

The retinae were examined with a bright ophthalmoscope using a green filter to obtain red-free illumination. Fundus photography was performed with the Zeiss Oberkochen fundus camera at $\times 2$ magnification. The fundus was recorded directly on Ilford $\mathrm{FP}_{4}$ black-and-white film, using a Wratten No. 65 (green) filter. Prints were made with high contrast filters on Kodak bromide paper.

\section{Right eye}

The temporal disc was pale with loss of capillaries and nerve fibre detail indicating diminished axons in the papillomacular bundle. A wrinkled appearance of superficial retinal light reflexes between the disc and macula, seen with the ophthalmoscope but not evident in photographs, was interpreted as wrinkling of the internal limiting membrane. The superior and inferior arcuate regions showed finely grained and interwoven linear striations of thickly packed nerve fibres that obscured the dark choroid beneath and dulled the light reflexes of small and large vessels. The arcuate nerve fibre bundles were best seen near the disc and gradually faded about three disc diameters peripherally (Fig. 1). 


\section{Left eye}

The temporal optic disc was pale and there was loss of nerve fibres in the papillomacular bundles. A patch of myelinated nerve fibres was contiguous with the superior temporal border of the disc (Fig. 2). Two parallel curvilinear grooves were seen in the superior arcuate nerve fibre bundle. These nerve fibre bundle defects extended proximally into the myelinated nerve fibres. The inferior defect extended closer to the disc, fading from view near its edge where adjacent nerve fibres converge. The upper defect formed a slit extending temporally for about two disc diameters. The defects were clearly distinguished from the fine feather-like pattern formed by normal nonmyelinated nerve fibres at the superior temporal margin of the myelinated area. Ophthalmoscopically they were dark green and devoid of the finely grained linear striations evident in the adjacent retina.

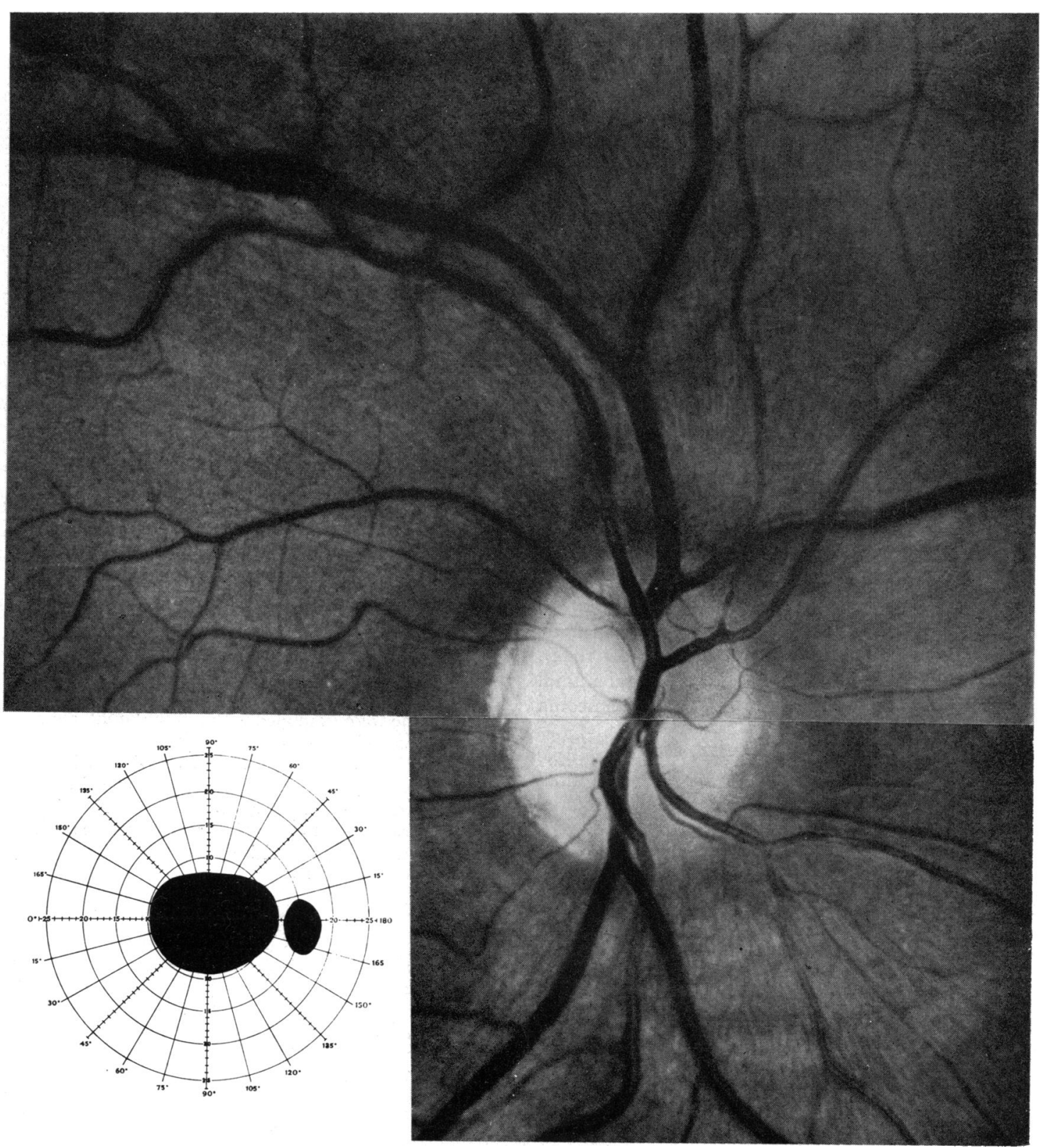

FIG. I Right fundus. The superior and inferior arcuate nerve fibre bundles show uniform striations and normal opacity. The nerve fibres obscure light reflexes of underlying blood vessels. The temporal disc is pale, and nerve fibre detail is not visible temporal to the disc. The visual field (inset) contains a dense central scotoma to a 50/2000 mm. white object 


\section{PERIMETRIC FINDINGS}

The visual fields were examined by kinetic perimetry using a $2 \mathrm{~m}$. Bjerrum screen and a Goldmann perimeter. Identical results were obtained at three separate examinations.
Right eye

There was a dense central scotoma to a $50 \mathrm{~mm}$. white stimulus. No arcuate defects were present and the peripheral isoptres were normal.

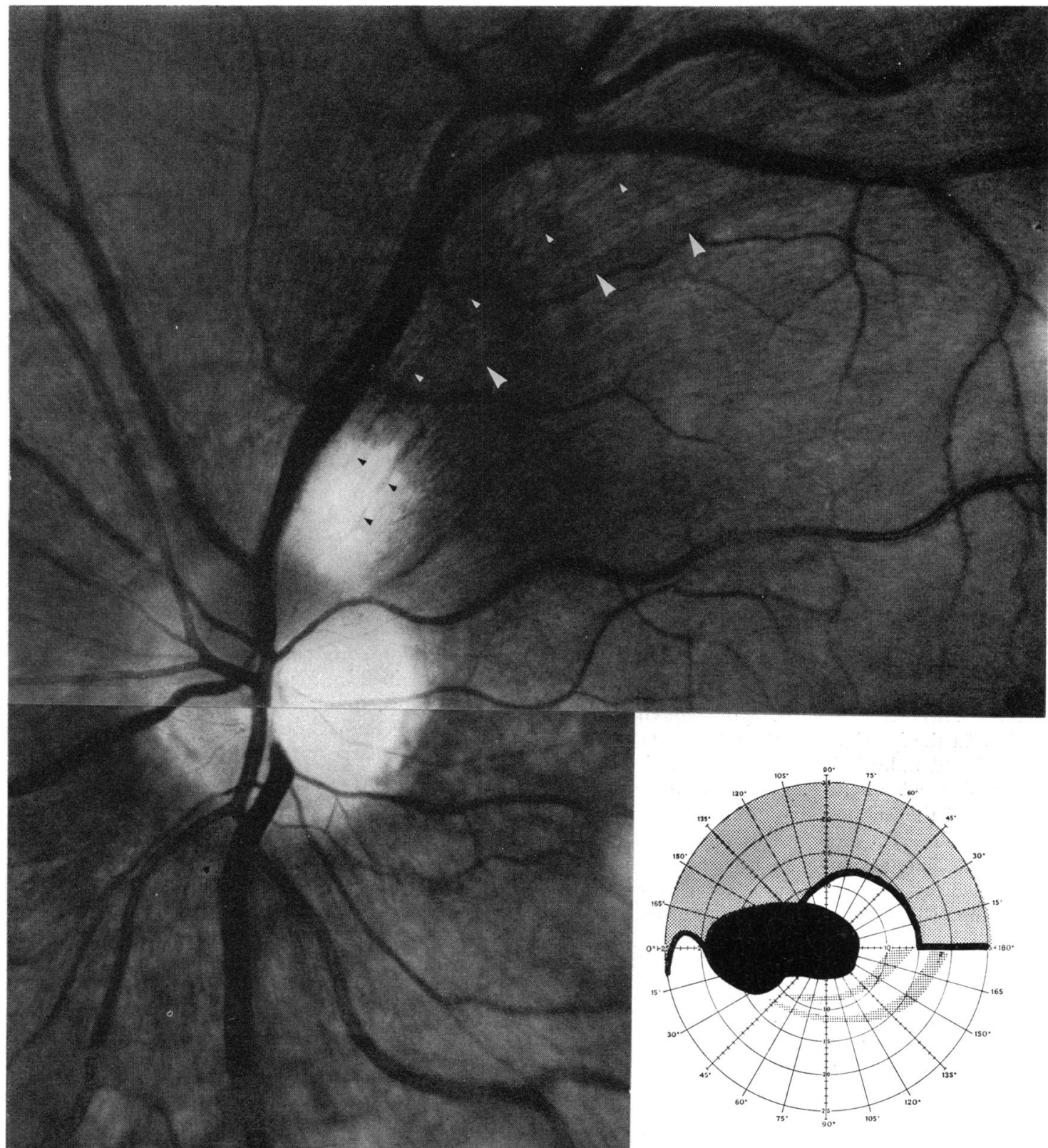

FIG. 2 Left fundus. Myelinated nerve fibres at the superior margin of the disc contain two dark linear defects (black arrows) continuous with defects in the superior arcuate bundle. The upper defect is a narrow slit (small arrows) and the lower defect forms a wedge (large white arrows). Nerve fibres are not visible temporal to the disc. The inferior arcuate bundle is thinned: nerve fibre striations are reduced and blood vessels exposed.

The visual field shows two inferior arcuate scotomata to a 2/2000 $\mathrm{mm}$. white stimulus and dense centrocaecal scotoma to a 50/2000 mm. white object. There is uniform depression of the upper Bjerrum area to a 2/2000 mm. white object. 
Left eye

Bjerrum screen examination demonstrated a dense centrocaecal scotoma to a $50 \mathrm{~mm}$. white object. The blind spot was enlarged inferonasally. The inferior Bjerrum area contained two arcuate defects to a $2 / 2000 \mathrm{~mm}$. white object. These scotomata were approximately $3^{\circ}$ wide nasally in the Bjerrum area and became narrower as they approached the blind spot. The patient reported that a $5 / 2000 \mathrm{~mm}$. white stimulus flickered off and on when passed slowly through the nasal portion of these scotomata. There was uniform depression of the upper arcuate field to a $2 / 2000 \mathrm{~mm}$. white object. Goldmann perimetry revealed normal peripheral isoptres. The $\mathrm{I}_{3}$ and $\mathrm{I}_{4}$ stimuli flickered when passed through the arcuate defects detected on the Bjerrum screen (Fig. I).

\section{Discussion}

Atrophy of nerve fibres was visible in the retina of our patient with optic neuritis. The linear defects in the myelinated nerve fibres were continuous with arcuate nerve fibre bundle defects, and signified demyelination in the retina.

Fundoscopic evidence of nerve fibre atrophy correlates well with visual deficits identified by perimetry (Hoyt and others, 1973). The signs of nerve fibre loss in our patient corresponded with loss of visual function determined by visual field examination. In the left eye, two inferior arcuate scotomata corresponded with two superior nerve fibre bundle defects. They were most easily detected on the Bjerrum screen. We could not predict the size or density of the scotomata from the appearance of the arcuate nerve fibre defects. The upper defect formed a narrow slit and the lower a wedge, but the scotomata were similar. In the inferior peripapillary retina, reduced nerve fibre detail, diminished opacity, and increased clarity of vascular light reflexes were signs of diffuse thinning of the inferior arcuate nerve fibre bundle. These features are most apparent when compared with the same region in the right fundus. The corresponding upper Bjerrum area showed mild depression. Reduced nerve fibre striations between the macula and disc corresponded to the centrocaecal scotoma, and the medullated nerve fibres produced enlargement of the blind spot.

In the right eye absence of visible nerve fibre detail at the temporal margin of the disc corresponded with the central scotoma. Wrinkling of the internal limiting membrane in this region, visible ophthalmoscopically, may be explained by diminution of the underlying nerve fibres.

Primary demyelination is the pathological substrate of multiple sclerosis. It is improbable that the retinal myelin in this case was affected by primary demyelination. Plaques of demyelination in multiple sclerosis are rounded or oval, often irregular in shape, and do not conform to the linear orientation of nerve fibres. Although primary demyelination tends to preserve axons (Peters, I968), axonal degeneration may be extensive (Adams and Kubik, 1952). Nerve fibre loss in the retina, as identified in our case, is a feature of multiple sclerosis (Frisén and Hoyt, 1975). We attribute the linear myelin loss in our patient to demyelination, secondary to retrograde degeneration of axons in the arcuate nerve fibre bundle. These myelin defects are a visible manifestation of secondary demyelination in the anterior visual pathway.

\section{Summary}

Atrophy of the nerve fibres in the retina visible by direct ophthalmoscopy using red-free illumination, together with corresponding scotomata in the visual fields, signified retrograde degeneration of retinal nerve fibres from multiple sclerosis. Defects in medullated retinal nerve fibres were continuous with arcuate nerve fibre bundle defects. These myelin defects exemplify secondary demyelination of optic axons.

We are indebted to Dr. W. Gooddy for allowing us to study this case. We thank Mr. R. Sennhenn, and the Department of Medical Illustration, and Miss G. Hopkins for secretarial assistance.

\section{References}

ADAMS, R. D., and KUBIK, c. s. (1952) Amer. F. Med., 12, 5 10

bachmanN, R. (1922) v. Graefes Arch. Ophthal., ro7, ro

FRISÉN, L., and HOYT, W. F. (1975) Arch. Ophthal., 92, 91

FUCHS, A. (195I) "Diseases of the Fundus Oculi", p. I4. Lewis, London

HOYT, W. F., FRISÉN, L., and NEWMAN, N. M. (1973) Invest. Ophthal., 12, 814

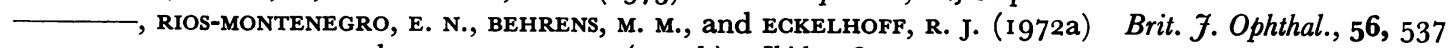

-, SCHLICKE, B., and EGKELHOFF, R. J. (1972b) Ibid., 56, 577

ITO, H., ozAWA, K., SUGA, s., and mizuno, K. (1969) Folia ophthal. jap., 20, 282

PETERs, G. (1968) In "Pathology of the Nervous System", vol. r, ed. J. Minckler, p. 82 r. McGraw-Hill,

New York

SACHSAlBER, A. (1905) Z. Augenheilk., 13, 739

vogt, A. (1913) v. Graefes Arch. Ophthal., 84, 293

(1921) Klin. Mbl. Augenheilk., 58, 399

wagenmann, A. (1894) v. Graefes Arch. Ophthal., 40, 250 